\title{
PROGRESSIVE MULTIFOCAL LEUKOENCEPHALOPATHY IN A KNOWN THYMOMA PATIENT
}

\author{
Amun Iqbal, Muhammad A. Naveed \\ Department of Radiology, Shaukat Khanum Memorial Cancer Hospital and Research Centre, Lahore, Pakistan \\ Received: 2 August 2018 / Accepted: 22 September 2018
}

A 48-year-old male with a previous history of thymectomy for thymoma, on intravenous immunoglobulin therapy, in remission, presented in emergency room with new onset of forgetfulness, disorientation and altered behaviour for 2 weeks. The blood investigations were significant for low lymphocyte count $0.9 \mathrm{U} / \mathrm{uL} \times 1000 \mathrm{U} / \mathrm{uL}$. Computed tomography (CT) brain was performed to evaluate for possible metastasis or ischaemia.

The CT brain revealed a large confluent non-enhancing hypoattenuation region in the left frontal lobe, centrum semiovale and external capsule, involving the subcortical and deep white matter with sparing of the overlying cortex, also extending to the contralateral right frontal lobe across the genu of the corpus callosum [Figure 1]. No associated mass effect or contrast enhancement was visualized. CT findings were suggestive of white matter disease such as demyelinating disease and further characterization with magnetic resonance imaging (MRI) was advised.

The MRI brain confirmed the findings of CT with confluent T2/fluid-attenuated inversion recovery (FLAIR) increased signal intensity in the left frontal lobe, involving the subcortical and deep white matter with sparing of the cortex, tracking along the genu of the corpus callosum to the contralateral right frontal lobe. There was involvement of subcortical U-fibres [Figure 2]. No mass effect, diffusion restriction or post-contrast enhancement were noticed [Figures 3 and 4]. Imaging findings were in favour of pure white matter disease such as progressive multifocal leukoencephalopathy (PML) in a patient with deficient cell-mediated immunity.

Other diseases can give similar appearance on imaging in immunocompromised patients such as immune

Correspondence: Dr. Amun Iqbal, Department of Radiology, Shaukat Khanum Memorial Cancer Hospital and Research Centre, Lahore, Pakistan.

Email: amykame04@gmail.com reconstitution inflammatory syndrome (IRIS) which can show intense irregular rim enhancement and diffusion restriction at the periphery. ${ }^{[1]}$ IRIS is seen in HIV-infected patients and patients getting PML-inducing immune therapy. ${ }^{[2]}$

Toxoplasmosis demonstrates ring enhancement and mass effect in the periventricular location. Cytomegalovirus infection can show ependymitis or ventriculitis. ${ }^{[1]}$ HIV encephalopathy has more confluent
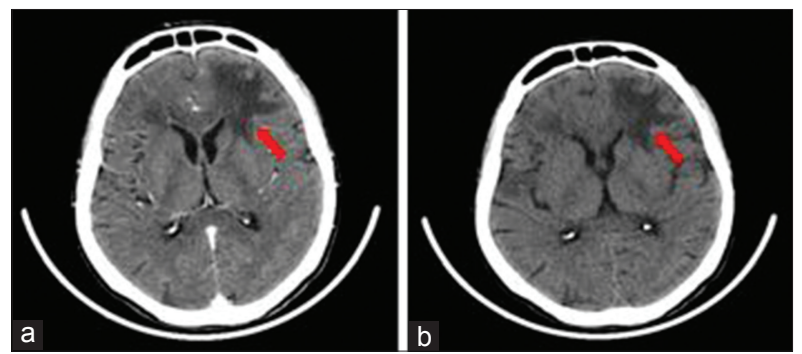

Figure 1: ( $\mathrm{a}$ and b) Axial enhanced and unenhanced computed tomography images of the brain show hypodense region in the left frontal lobe subcortical and periventricular white matter (red arrow)

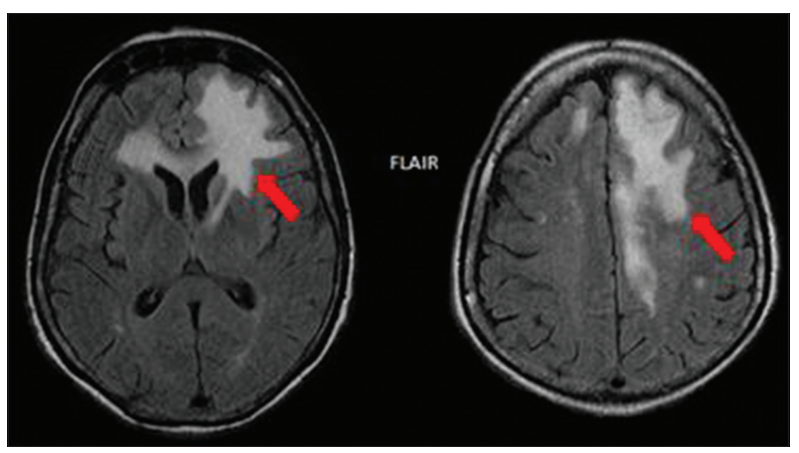

Figure 2: Axial fluid-attenuated inversion recovery magnetic resonance images demonstrate confluent high-signal intensity region in the left frontal lobe involving the subcortical and deep white matter, extending to the left external capsule left centrum semiovale and right frontal lobe across the genu of the corpus callosum 


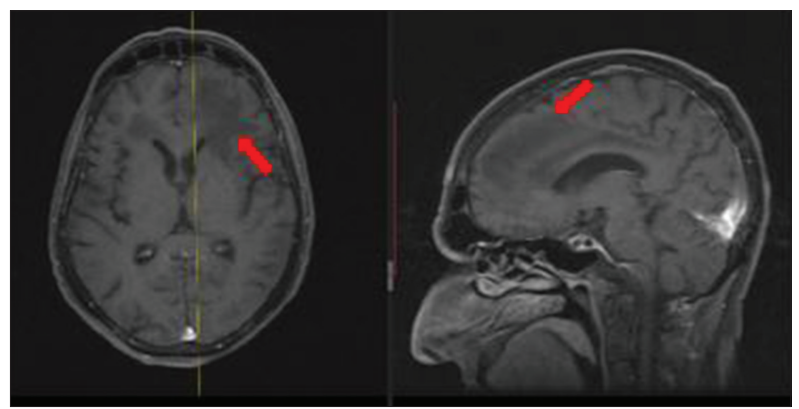

Figure 3: Axial and sagittal T1 post-gadolinium magnetic resonance images show no contrast enhancement or mass effect

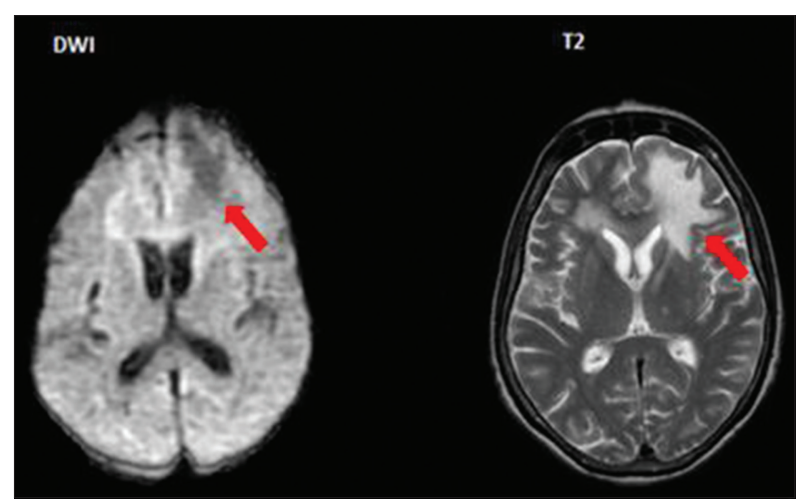

Figure 4: Axial diffusion-weighted image magnetic resonance image shows no diffusion restriction

symmetric periventricular distribution associated with brain atrophy.

PML is a disease involving central nervous system when there is the destruction of oligodendrocytes by John Cunningham (JC) virus. ${ }^{[3]}$ It is a type of opportunistic infection in immunocompromised patients such as HIV infection, lymphomas, organ/stem cell transplantation and patients who are subjected to immune therapies with monoclonal antibodies like natalizumab.

PML classically shows T1 hypointense and T2/FLAIR hyperintense asymmetric multifocal periventricular and subcortical white matter involvement on MRI. It has predilection for parietooccipital areas with more common involvement of subcortical U-fibres. No mass effect or contrast enhancement is observed and cortex is usually spared. PML-IRIS, a similar disease seen in AIDS patients on HAART or natalizumab therapy, there is post-contrast enhancement in these white matter changes. It may be considered as a sign of better survival. Diffusion-weighted image sequences show patchy diffusion restriction in the periphery of these white matter changes.

Definitive diagnosis of PML is based on histopathology which includes findings such as demyelination, bizarre astrocytes, enlarged oligodendroglial nuclei and presence of JC virus. Another diagnostic tool is the presence of JC virus in cerebrospinal fluid (CSF). It is detected by PCR. A number of authorities regard the demonstration of JC viral DNA in CSF coupled with the appropriate clinical and radiologic features, sufficient to diagnose PML, thus obviating the need for brain biopsy. ${ }^{[4]}$ Therefore, clinical, radiologic and laboratory evidence are essential to establish diagnosis.

\section{Conflict of Interest}

The authors declare that they have no conflict of interest.

\section{References}

1. Osborn AG. Osborn's Brain: Imaging, Pathology, and Anatomy. Salt Lake City, Utah: Amirsys Publication; 2013.

2. Smith AB, Smirniotopoulos JG, Rushing EJ. From the archives of the AFIP: Central nervous system infections associated with human immunodeficiency virus infection: Radiologicpathologic correlation. Radiographics 2008;28:2033-58.

3. Grossman RI, Yousem DM. Neuroradiology, the Requisites. $2^{\text {nd }}$ ed. Philadelphia, PA: Mosby Inc.; 2003.

4. Cinque P, Koralnik IJ, Clifford DB. The evolving face of human immunodeficiency virus-related progressive multifocal leukoencephalopathy: Defining a consensus terminology. J Neurovirol 2003;9 Suppl 1:88-92. 